\title{
Computer Vision for Interactive Skewed Video Projection
}

\author{
Alessandro Brazzini and Carlo Colombo \\ Dipartimento di Sistemi e Informatica, \\ Via Santa Marta 3, I-50139 Firenze, Italy \\ colombo@dsi.unifi.it
}

\begin{abstract}
We present an uncalibrated projector-camera system in which the information displayed onto a planar screen can be interactively warped according to an arbitrary planar homography. The user interacts with the system through a laser pointer, whose displacements on the screen plane are captured by the camera, interpreted as mouse drags, and used to control the warping process. Applications of our interactive warping system encompass arbitrary (pan/tilt/screw) keystone correction, visualization of undistorted information for a user being in a general position with respect to the screen (including virtual anamorphosis as a special case), and self-shadow avoidance by a nearly-parallel projection.
\end{abstract}

\section{Introduction}

Presentation systems based on computer-controlled video projection have recently become a de facto standard in almost every field of professional activity, ranging from business to science and education. Indeed, the digital representation of audio-visual material allows a uniform and highly flexible software treatment of multimedia information, and makes it possible to replace media-specialized display devices as analog VCR's and slide projectors with a single, general purpose digital projector. Projector-camera systems are one of the latest evolutions of the research in the field. These systems use one or more cameras to provide the computer with visual feedback about the presentation scenario, and specifically (1) the information displayed on the screen and/or (2) the human user's activity.

Feedback of type (1) was recently used to automatically compensate for the so called "keystone" deformation. This is a projective deformation of the original display area arising in the presence of misalignments between the projector and the screen planes. Current projectors normally include a hardware keystone correction, but this is typically limited to a 1-dof (tilt) misalignment. The keystone can be represented in the most general way as a planar homography mapping points in the projector plane onto the screen plane, corresponding to a 3 -dof misalignment (pan, tilt, screw). To eliminate the effect of the keystone, its associated homography can be estimated and used to suitably pre-deform the image being displayed. Several methods were proposed recently to estimate the keystone homography - without any knowledge of either projector and camera calibration 
parameters - through the knowledge of the coordinates of some reference points on the screen plane. In particular, in [7, the wall used for display is augmented with four fiducial markers arranged according to a known pattern; similarly, in [1], planar objects with standard shape such as postcards are used; finally, in [8], a blank screen with known shape is used.

Feedback of type (2) was also recently exploited in several fashions in order to improve the way users interact with the system. A general topic of research in this field, actually not limited only to presentation systems, is that of the development of human-computer interaction devices based on the visual interpretation of user motions and gestures, with the purpose of replacing the conventional mouse and keyboard with more natural and effective pointing systems (see [2] for a review). Another research topic, more closely related to the design of camera-projector systems, exploits locating inside the displayed area the laser spot normally used during presentations, so as to infer user intentions and convert them into interface commands. Recent research in this field was focused on using laser pointers so as to simulate mouse clicks, and select interface buttons through a temporal analysis of laser spot changes [4, 5], 8].

In this paper, feedback information of both types (1) and (2) is used so as to design an uncalibrated projector-camera system in which the information displayed onto a planar screen can be interactively warped using a laser pointer according to an arbitrary planar homography. The continuous movements of the laser spot on the screen plane are captured by the camera, interpreted as mouse drags, and used to control the warping process. We demonstrate the usefulness of our interactive warping system (IWS) for several applications, ranging from semi-automatic general keystone correction (not requiring any reference screen points), to visualization of undistorted information for users in a general position with respect to the screen (including virtual anamorphosis as a special case), and self-shadow avoidance by a nearly-parallel projection. Experimental results in terms of task completion time and user satisfaction show that the system is suitable for real application scenarios. Video materials are at the page wWW.dsi.unifi.it/users/colombo/research/IWS/IWS.zip.

\section{Interactive Warping System}

\subsection{Overview}

Figure 1 shows the main elements of the system. The user (U), projector $(\mathrm{P})$, camera $(\mathrm{C})$, and screen $(\mathrm{S})$ are located in general position with respect to each other. Let $I_{d}$ be a PC image being displayed, and $I_{u}(t)$ the same image as perceived by the user at time $t$. The goal of the system is to cooperate with the user so as to let him gradually change the appearance of $I_{u}(t)$ to obtain a goal image $I_{g}$. If the goal image is equal to $I_{d}$, then the user task is to compensate for all the geometric and optical distortions introduced by the system elements. This leads to the application scenarios of keystone correction and purposive misalignment addressed respectively in subsections 3.1 and 3.2 . If $I_{g} \neq I_{d}$, then the task is that of arbitrary viewpoint change, discussed in subsection 3.3 . 


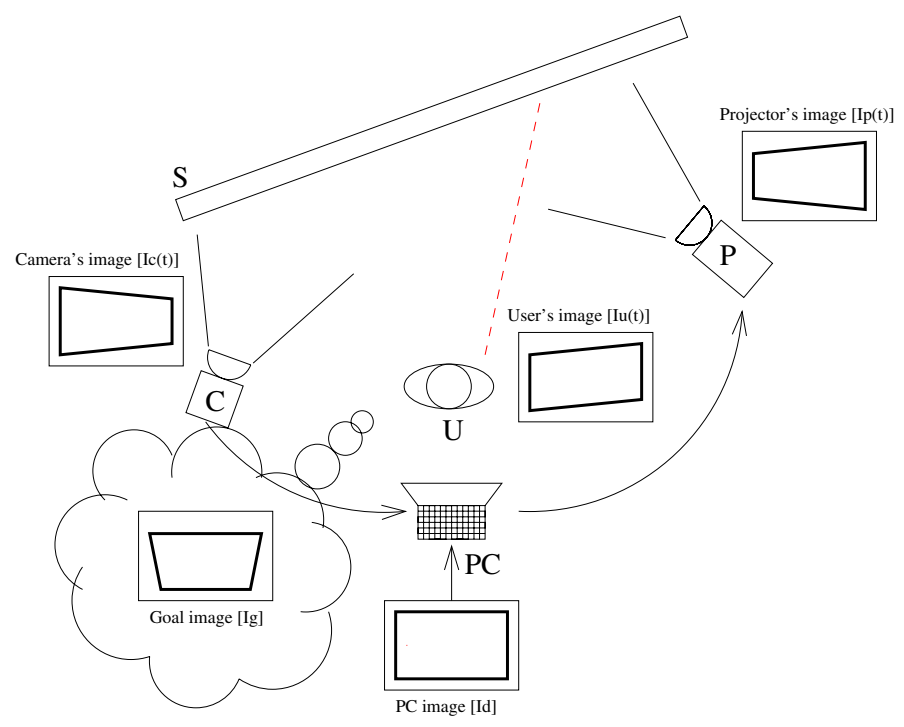

Fig. 1. The elements of the system

\subsection{Modeling and Implementation}

Mathematically speaking, the different tasks mentioned above can be uniformly described as instances of a general system objective, i.e. to iteratively deform $I_{d} \in \mathbb{P}^{2}$ into the projected image $I_{p}(t)$ according to a time-varying planar homography $H_{d}(t): I_{d} \rightarrow I_{p}(t)$, until $I_{u}(t)=I_{g}$ up to a scale parameter.

The graph of Fig. 2] summarizes the geometric planar-projective relationships (graph edges) between pairs of system elements (graph nodes). Notice that any change to $H_{d}(t)$ induces a change in all of the images but $I_{d}$ (and, of course, $I_{g}$ ).

The homography $H_{c}(t): I_{c}(t) \rightarrow I_{p}(t)$ relates the camera and projector images. If both projector and camera are assumed to be fixed, and to have unknown

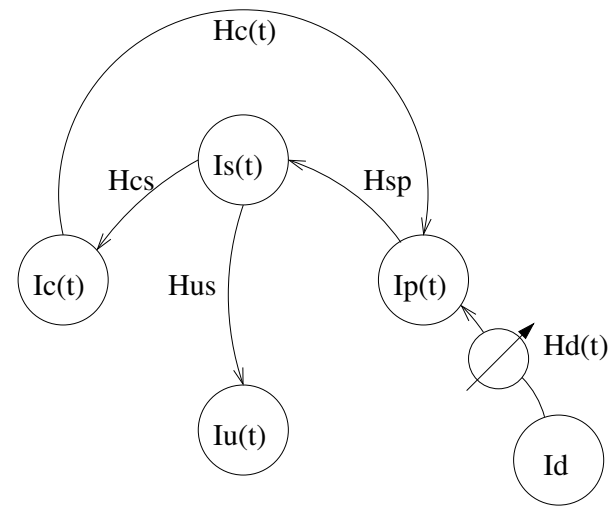

Fig. 2. Geometric relationships between system elements 
but constant internal parameters, then $H_{c}(t)$ is actually a time-invariant transformation $H_{c}$, that can be easily computed once and for all at system startup from four or more point correspondences as shown in [3]. The deformation homography $H_{d}(t)$ is obtained for each $t$ as follows. As the user points at the screen with his laser beam, the position of the laser spot on $\mathrm{S}$ is imaged by $\mathrm{C}$ at $\mathbf{x}_{c}(t)$. The point $\mathbf{x}_{p}(t)$ on the projector's plane can be consequently computed as $\mathbf{x}_{p}(t)=H_{c} \mathbf{x}_{c}(t)$. Now, consider the four corners $\mathbf{a}, \mathbf{b}, \mathbf{c}, \mathbf{d}$ of the image $I_{p}(t-1)$, and assume without loss of generality that $\mathbf{b}$ is the one closest to $\mathbf{x}_{p}(t)$. Then, $H_{d}(t)$ is computed as the transformation leaving unaltered $\mathbf{a}, \mathbf{c}$ and $\mathbf{d}$, and replacing $\mathbf{b}$ with $\mathbf{x}_{p}(t)$. On the user's side, the overall effect is to perceive to be using the laser beam as a means to control the position of $\mathbf{b}$.

The laser spot is localized at each frame on the image plane by a simple red spot detector based on color and intensity thresholding. For the sake of temporal smoothing, the located spot $\mathbf{x}_{c}(t)$ is obtained as the output of a mobile mean filter with constant gain $\alpha=0.2$.

\section{Applications and Results}

\subsection{Semi-automatic Keystone Correction}

Fig. 3 shows the geometry for keystone correction. In Fig. 3 (left) is depicted the special case of keystone correction where the user is located in a frontoparallel way with respect to the screen. This case is the only case practically addressed in an embedded way by modern video projectors. Similarly, all of the techniques for automatic keystone correction proposed so far in the literature and described in the introduction address only this special case.

The general case of keystone correction, which can be correctly handled by IWS, is shown in Fig. 3 (right). In this case, the user is in general position with respect to the screen, and yet is able to compensate for the distortions induced by the system.

Fig. 4 illustrates the process of keystone correction.The figure also shows the laser spot and one of the four interest regions around the current image corners in which the spot is searched for. Notice that, thanks to its "what you
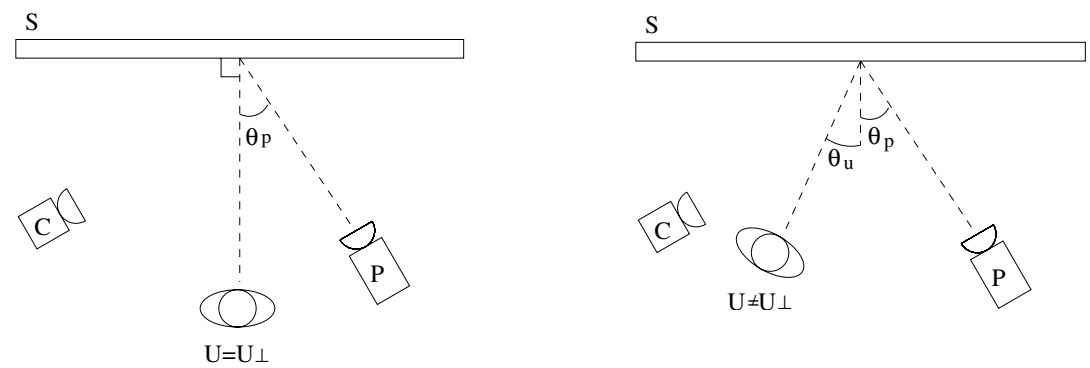

Fig. 3. (Left) Standard keystone correction (frontoparallel user). (Right) Extended keystone correction (user in general position). 

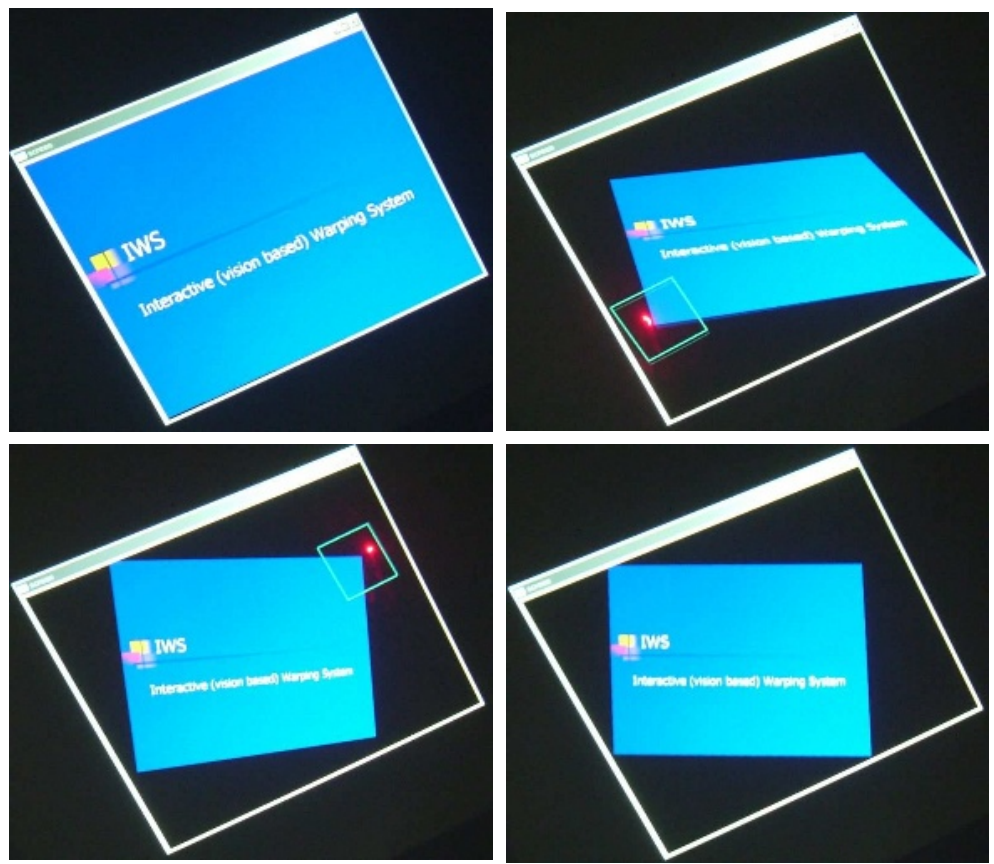

Fig. 4. Semi-automatic keystone correction. (Upper Left) Initial view. (Upper Right), (Lower Left) Two intermediate phases. (Lower Right) Final view.

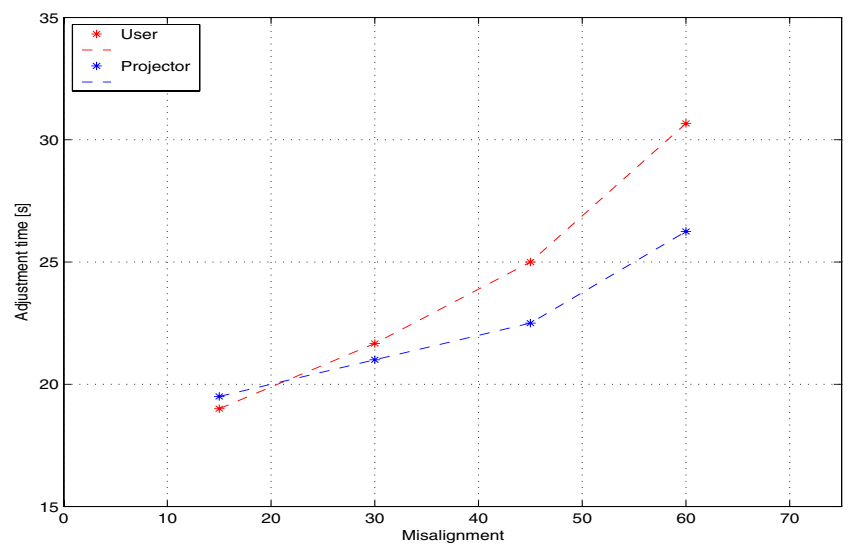

Fig. 5. Task completion time performance for varying projector angles $\theta_{p}\left(\theta_{u}=0\right)$ and user angles $\left(\theta_{p}=\pi / 6\right)$

see is what you get" nature, the homography-based approach allows users to cope with general pan/tilt/screw keystone misalignments in a very effective and natural way: The same could not be obtained by three distinct buttons, each controlling a separate dof. 
System performance in terms of task completion time for a group of seven different users and a general 3-dof misalignment is shown in Fig. 5. The time required to adjust the keystone slowly and linearly increases as the misalignment angles $\theta_{p}$ (projector) and $\theta_{u}$ (user) with respect to the screen normal increase. A completion time of about $30 \mathrm{~s}$ is required for misalignment angles of about 60 degrees. This is not a penalizing performance, also considering the fact that the keystone correction has to be performed at system startup and has not to be repeated very frequently. System performance appears to be slightly worse for user misalignments than for projector misalignments, possibly due to the fact that in the latter case users can often rely on fiducial lines (e.g., the line between the floor and the screen wall) for the completion of their task.

\subsection{Purposive Misalignments}

The geometric configuration (Fig. 6) for purposive misalignment is similar to that of Fig. 3, but the application domain is different. In fact, the figure shows
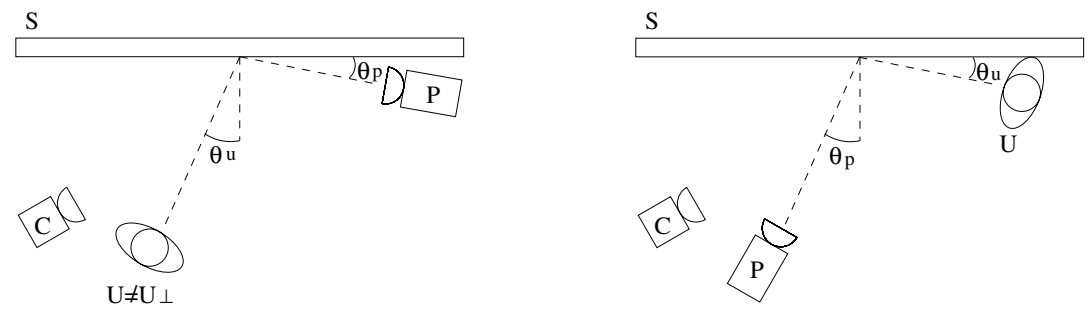

Fig. 6. (Left) Self-shadow avoidance. (Right) Virtual anamorphosis.

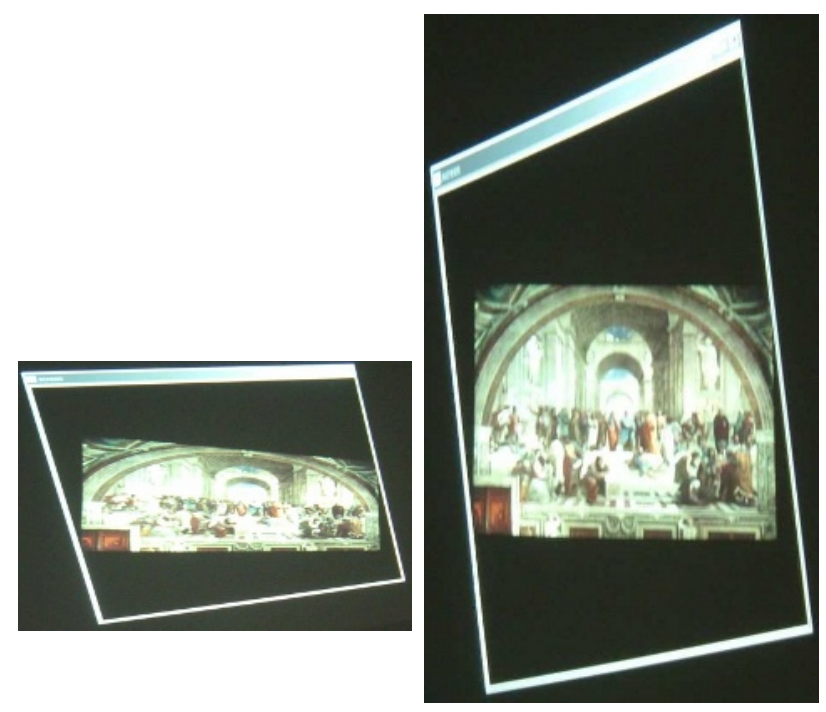

Fig. 7. Virtual anamorphosis. (Left) Frontoparallel view. (Right) Slanted view. 
on the left the case in which the projector is slanted as much as possible with respect to the screen, so as to avoid the phenomenon of user self-shadowing and dazzling: This can be obtained without the need of expensive and cumbersome equipment such as that required for rear video projection.

On the right is reported instead the case of virtual anamorphosis, where the image $I_{p}$ is very much deformed, and is correctly intelligible only for users observing the screen plane from a slanted viewpoint. Indeed, virtual anamorphosis is a means to convey significant visual information only to a restricted number of people around a selected viewpoint; as such, it is a way to implement a sort of directional vision as the analogous of directional audio. A typical operational scenario for virtual anamorphosis is when the image is projected onto the pavement or the ceiling of a room (see Fig. 7).

\subsection{Arbitrary Viewpoint Change}

Fig. 8 illustrates the geometry for arbitrary viewpoint change. In this scenario, the user can look at the image of a $3 \mathrm{D}$ object from a viewpoint that can greatly differ from the projection center used to obtain the image.

A possible application is in the architectural domain, where the photograph of the façade of a building taken from a non frontoparallel viewpoint can be interactively rectified so as to eliminate perspective distortions. Another inter-

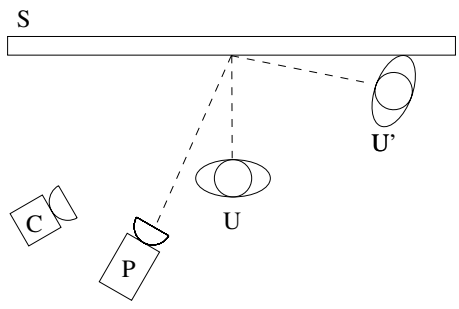

Fig. 8. Arbitrary viewpoint change: Geometry
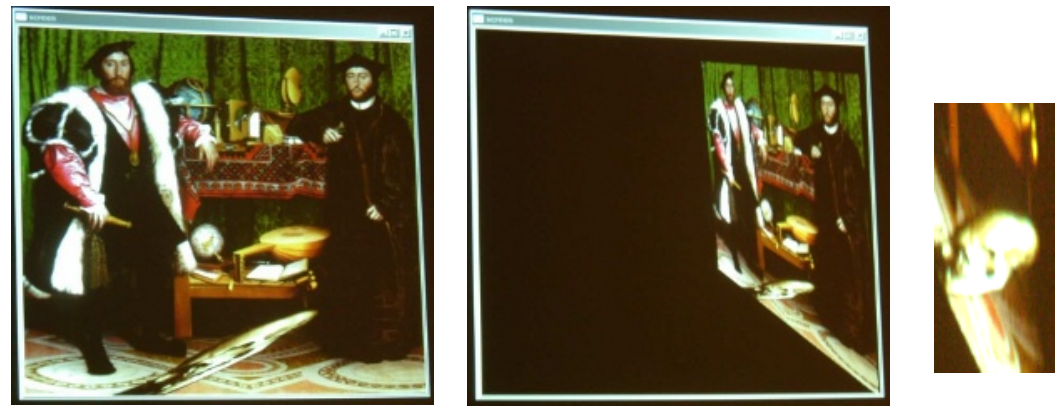

Fig. 9. Arbitrary viewpoint change. (Left) Initial (frontoparallel) view. (Middle) Final (anamorphic) view. (Right) Particular of the skull. 
esting application is in the software-controlled visualization of paintings. For instance, Fig. 9 shows arbitrary viewpont change applied to the painting "The ambassadors" by Hans Holbein. The new viewpoint selected corresponds to the auxiliary viewpoint chosen by the artist to hide an anamorphic enigma inside his painting. The enigma is solved by a suitable interactive warping of the original painting.

\section{References}

1. M. Ashdown, M. Flagg, R. Sukthankar, and J. Rehg, "A flexible projector-camera system for multi-planar displays." In Proc. IEEE Int. Conf. on Computer Vision and Pattern Recognition (CVPR), pp. II:165-172, IEEE 2004.

2. C. Colombo, A. Del Bimbo and A. Valli, "Visual capture and understanding of hand pointing actions in a 3D environment." IEEE Trans. on SMC(B), 33(4):677686, IEEE 2003.

3. R. I. Hartley and A. Zisserman. Multiple View Geometry in Computer Vision. Cambridge University Press, 2000.

4. C. Kirstein and H. Muller, "Interaction with a projection screen using a cameratracked laser pointer." In Proc. Int. Conf. on Multimedia Modeling (MMM), pp. 191192, IEEE 1998.

5. D. Olsen Jr. and T. Nielsen, "Laser pointer interaction," in Proc. ACM SIGCHI Conf. on Human Factors in Computing Systems, pp. 17-22, ACM 2001.

6. R. Raskar and P. Beardsley, "A self correcting projector," In Proc. IEEE Int. Conf. on Computer Vision and Pattern Recognition (CVPR), pp. 504-508, 2001.

7. J. M. Rehg, M. Flagg, T.-J. Cham, R. Sukthankar, and G. Sukthankar, "Projected light displays using visual feed-back." In Proc. 7th Int. Conf. on Control, Automation, Robotics and Vision (ICARCV), pp. II:926-932, 2002.

8. R. Sukthankar, R. G. Stockton, and M. D. Mullin, "Smarter presentations: exploiting homography in camera-projector systems." In Proc. IEEE Int. Conf. on Computer Vision (ICCV), pp. 247-253, IEEE 2001. 